\title{
Programmed Acute Electrical Stimulation of Ventral Tegmental Area Alleviates Depressive-Like Behavior
}

\author{
Alexander Friedman', Michael Frankel ${ }^{2}$, Yakov Flaumenhaft ${ }^{2}$, Avia Merenlender ${ }^{2}$, Albert Pinhasov ${ }^{3}$, \\ Yuval Feder ${ }^{3}$, Michal Taler', Irit Gil-Ad' ${ }^{4}$, Moshe Abeles' and Gal Yadid*, I,2 \\ 'Leslie and Susan Gonda (Goldschmied) Multidisciplinary Brain Research Center, Bar-llan University, Ramat Gan, Israel; ${ }^{2}$ The Mina and Everard \\ Goodman Faculty of Life Sciences, Bar-llan University, Ramat Gan, Israel; ${ }^{3}$ Department of Molecular Biology, Ariel University Center of Samaria \\ Ariel, Ariel, Israel; ${ }^{4}$ Laboratory of Biological Psychiatry, Felsenstein Medical Research Center, Sackler Faculty of Medicine, Tel-Aviv University, \\ Tel-Aviv, Israel
}

Depressive disorders affect approximately $5 \%$ of the population in any given year. Antidepressants may require several weeks to produce their clinical effects. Despite progress being made in this area there is still room and a need to explore additional therapeutic modes to increase treatment effectiveness and responsiveness. Herein, we examined a new method for intervention in depressive states based on deep brain stimulation of the ventral tegmental area (VTA) as a source of incentive motivation and hedonia, in comparison to chemical antidepressants. The pattern of stimulation was fashioned to mimic the firing pattern of VTA neurons in the normal rat. Behavioral manifestations of depression were then monitored weekly using a battery of behavioral tests. The results suggest that treatment with programmed acute electrical stimulation of the VTA substantially alleviates depressive behavior, as compared to chemical antidepressants or electroconvulsive therapy, both in onset time and longitudinal effect. These results were also highly correlated with increases in brainderived neurotrophic factor mRNA levels in the prefrontal cortex.

Neuropsychopharmacology (2009) 34, 1057-1066; doi:I0.1038/npp.2008.177; published online 8 October 2008

Keywords: depression; deep brain stimulation; ventral tegmental area; reward; BDNF

\section{INTRODUCTION}

Depressive disorders affect approximately $5 \%$ of the population in any given year $(6.5 \%$ of women and $3.3 \%$ of men). Approximately $5-12 \%$ of men and $10-25 \%$ of women will suffer from depression at any point in life. Over $45 \%$ of patients exhibit relapse to depression after responding to the available treatments. In light of the wide spread nature of this disorder there and its severity, there seems to be a genuine need for the development and improvement of antidepressant treatments (Kessler et al, 2003).

Electrical deep brain stimulation (DBS), a technique used to treat a number of neurological disorders, is an effective method found to significantly alleviate symptoms of Parkinson's disease, dystonia, and Tourette's syndrome. Recent studies reported improved response in resistant depressive patients (66\%), after DBS of the white matter tracts adjacent to the subgenual cingulate gyrus (SUC;

*Correspondence: Dr G Yadid, The Mina and Everard Goodman Faculty of Life Sciences and Leslie and Susan Gonda (Goldschmied) Multidisciplinary Brain Research Center, Bar-llan University, Geha Road, Ramat-Gan 52900, Israel. Tel: +972 3 531 8123,

Fax: + 9723635 4965, E-mail: Yadidg@mail.biu.ac.il

Received 27 May 2008; revised 6 August 2008; accepted 8 August 2008
Mayberg et al, 2005), and in the nucleus accumbens (N.Ac; Schlaepfer et al, 2008). However, continuous intensive stimulation at a relatively high frequency was needed.

Brain-derived neurotrophic factor (BDNF) was originally identified as having neurotrophic activity (Barde et al, 1982). Several experiments have suggested a functional role for BDNF in mediating antidepressant effects. BDNF expression is increased by chronic, but not acute, administration of several antidepressant drugs (tranylcypromine, sertraline, desipramine, or mianserin) (Dias et al, 2003; Nibuya et al, 1995; Thome et al, 2000). Similarly, evidence has implicated BDNF in the effect of electroconvulsive treatment (ECT) and chronic electrical convulsive stimulation on depressive behavior (Dias et al, 2003; Nibuya et al, 1995).

There are a number of sources of evidence that indicate the involvement of the dopaminergic system in the mechanisms underlying depression (Gershon et al, 2007). One source of evidence demonstrates that depressed patients show a decrease in dopamine (DA) neurotransmission. Furthermore, it has been found in studies of depressed patients that there exists a reduction in the cerebrospinal fluid levels of the DA metabolite, homovanillic acid (Kapur and Mann, 1992; Reddy et al, 1992). Additional support comes from the finding that there is an 
association between alterations in DA levels achieved by pharmacological means and the level of depressive symptoms (Kapur and Mann, 1992; Ordway and Mann, 2002). A similar correlation has been found in the learned helplessness animal model of depression (Kapur and Mann, 1992).

A second source of evidence arises from exploring the dopaminergic system's pathways. Dopaminergic neurons in the ventral tegmental area (VTA) of the midbrain project to limbic areas including the N.Acs, olfactory tubercle, and septum, and to cortical areas, including the cingulate, prefrontal, entorhinal, and pyriform cortices. Findings from animal studies have indicated these pathways as mediators of reward-driven, pleasurable behavior (Naranjo et al, 2001). Detrimental changes in such behavior, shown by depressed individuals further strengthen the case for the involvement of the dompaminergic system.

We previously described (Dremencov et al, 2004, 2005, 2006) in this specific animal model of depression, that the activity of the mesolimbic dopaminergic system is critical for responsiveness to antidepressant treatments. The VTA is the origin of dopaminergic neuron cell bodies in the mesolimbic system and as such presents itself as a reasonable site for intervention through DBS. Further support for this choice comes from the previously mentioned study (Mayberg et al, 2005), which demonstrated that DBS of white matter tracts adjacent to the SUC, which projects to the VTA, and which as a result of the SUC stimulation was subsequently activated, can elevate depressive symptoms.

In a previous study, we were able to decode and identify specific patterns of activity associated with depressive-like behavior of Flinder Sensitive Line (FSL) rats, a genetic animal model of depression (Overstreet et al, 2005). We were able to demonstrate that the standard VTA firing pattern, of Sprague-Dawley (SD) rats, was composed of bursts containing a large amount of spikes. In contrast, when examining FSL rats, we rarely found such a firing pattern. In FSL rats we detected a significant alteration in burst-like and cluster activities that was restored by treatment with desipramine (Friedman et al, 2007).

In the present study, we tested a new method for intervention in depressive disorders, based on DBS of the VTA. We used acute electrical stimulation (AES), a modified version of DBS, which applies short-term lowfrequency programmed stimulation instead of continual DBS. This method was tested in the FSL rats and was compared to conventional antidepressant treatments. FSL rats demonstrate characteristic depressive behavior, including reduced appetite and general activity, anhedonia, and loss of motivation. In addition, FSL rats exhibit positive response to various clinically used antidepressant therapies. The effect of AES on depressive-like behavioral manifestations was monitored by a battery of five behavioral tests. BDNF expression in different brain regions following AES was measured by real-time PCR and western blot analysis.

On the basis of our results, we suggest a substantial remedial effect of AES on depressive behavior, in addition to the functional role of BDNF in mediating the antidepressant effects of AES.

\section{MATERIALS AND METHODS}

\section{Animals}

The FSL partially resembles depressed individuals. These rats exhibit sleep and immune abnormalities, reduced appetite, and features of psychomotor function that are observed in depressed individuals. Neurochemical and pharmacological evidence suggests that FSL rats exhibit changes consistent with the cholinergic, serotonergic, dopaminergic, noradrenergic, and GABAergic models of depression. Therefore, this model is useful as a screen for antidepressants and their efficiency (Overstreet $e t$ al, 2005). Male (250-300 g) FSL rats as well as SD rats (as controls) were maintained under conditions of unvarying temperature $\left(25^{\circ} \mathrm{C}\right)$ and humidity $(50 \%)$, in a $12: 12 \mathrm{~h}$ light/dark cycle and with free access to food and water.

\section{In Vivo Electrophysiology Recording}

Rats were anesthetized with chloral hydrate $(400 \mathrm{mg} / \mathrm{kg}$, i.p.) and a recording electrode (glass-coated tungsten, $5 \mathrm{M} \Omega$ ) was stereotaxically inserted into a tract in the VTA (anterior 5.3, lateral 0.5, ventral $8.1 \mathrm{~mm}$ from bregma). The level of anesthesia was kept constant and a slow heart rate ( 300 beats per $\min$ ) was maintained, monitored by continuous electrocardiogram recordings. Single unit recordings from VTA neurons of SD and FSL rats $(n=12$ per each group, 2500 spike recordings) were performed using amplitude discrimination. Firing of DA-like cells was identified and quantified as described previously (Friedman et $a l, 2008)$ in accordance with the findings of Grace and Bunney (1984a, b). Following the last recording, each animal underwent electrolytic lesioning $(0.5 \mathrm{~mA}$ of DC current, $15 \mathrm{~s})$ to determine the location of the electrode tip. After transcardially perfusing the rats, their brains were removed and post-fixed in $10 \%$ formaldehyde. Frozen sections $(50 \mu \mathrm{m})$ were cut and examined under a microscope. Only data sets recorded from the VTA-verified placement of the electrode tip were analyzed.

\section{Electrode Construction and Surgical Procedures for AES}

Animals were anesthetized with ketamine hydrochloride $(100 \mathrm{mg} / \mathrm{kg}$, i.p.) and xylazine $(10 \mathrm{mg} / \mathrm{kg}$, i.p.) before stereotaxic surgery. A bipolar stimulating electrode (stainless steel, $0.08 \mathrm{~mm}$ diameter with cathode and anode isolation) was inserted unilaterally into the VTA (anterior 5.3 , lateral 0.5 , ventral $8.1 \mathrm{~mm}$ from bregma). We tested site specificity by stimulation in another region within close proximity to the VTA, the deep mesencephalic nucleus (anterior 5.3, lateral 1.5, ventral $6.6 \mathrm{~mm}$ from bregma). The implantation was secured to the skull with screws and dental acrylic cement. After surgery rimadyl ( $2 \mathrm{mg} / \mathrm{kg}$, i.p.) was administered.

\section{Stimulation Procedure}

The calculated pattern of bursts in SD rats was applied to the VTA of FSL rats, using DBS $(300 \mu \mathrm{A}$; the pattern of stimulation was composed of two bursts per second. Each burst contained five spikes with an interval of $80 \mathrm{~ms}$ between spikes, and with a $180 \mathrm{~ms}$ pause after each burst; 
in total, a stimulation of $10 \mathrm{HZ}$, see Supplementary Figure 1B). Stimulation was produced once in each rat, for a period of $20 \mathrm{~min}$.

\section{Monitoring Depressive-Like Behavior}

Swim test. Depressive-like behavior (despair) was measured by calculation of immobility time using a modified version of the Porsolt forced swim test (Dremencov et al, 2006; Overstreet, 1993; Overstreet et al, 2005). It is important to note that because our model is a genetic one, the swim test is not used to induce depressive behavior as in other models of depression. Rather, it serves as a test to examine the efficacy of various depressive treatments. In brief, the test is composed of a clear cylindrical tank $(40 \mathrm{~cm}$ high and $18 \mathrm{~cm}$ in diameter) containing enough water (at $2{ }^{\circ} \mathrm{C}$ higher than room temperature) to allow the rats to touch the bottom with their tail. Rats were considered to have stopped swimming when both hindpaws were immobile. Test duration was $5 \mathrm{~min}$.

Social behavior test. The behavior testing apparatus was based on a design by Malatynska and Kostowski (1984). The apparatus and procedure have been described elsewhere (Knapp et al, 2002; Malatynska et al, 2002). Briefly, the apparatus was composed of two chambers $(24 \times 17 \times 14 \mathrm{~cm})$ that were connected by a tunnel $(4.5 \times 4.5 \times 52 \mathrm{~cm})$ that due to its size permitted only one rat to pass at a time. A feeder containing sweetened milk ( $20 \%$ sucrose solution) was situated at the mid-point of the tunnel. Gated holes were constructed on both sides of the feeder to allow an equal start for the paired rats.

The same procedure, with modifications of the criteria, for the selection of dominant-submissive pairs of rats (Pinhasov et al, 2005a), was used by us (Pinhasov et al, 2005b).

In brief, dominant-submissive relationship testing begins with the random assignment of rats into pairs. These pairs were brought together once a day, during the testing period. Otherwise, they were housed separately with other animals in groups of three. Each member of a pair was placed in opposite chambers of the testing apparatus. The time spent on the feeder (milk with $20 \%$ sucrose) by each animal was recorded during a 5-min testing period. Then, the animals were separated, and returned to their home cages.

During the first 2 weeks (12 days) of testing, rats were initially habituated. Rats were tested before surgery (naive rats), 10 days after surgery (operated rats), after AES (FSL rats were treated by AES), and once per week, up to a month after AES. Animals had free access to water and food, thus the test measured only submissive behavior.

Locomotor activity test. Rats were initially habituated to an open field, a plastic polymer box $(90 \times 90 \times 30 \mathrm{~cm})$, for 5 min per day for 14 days before surgery. All measurements were performed between 0800 and 1400 hours in red light. A camera was placed above the box. The video and computer equipment was situated in a separate room, in which all video and observation analysis was conducted. On day 15, each rat was filmed in the open field for $5 \mathrm{~min}$, so that naive behavioral parameters could be measured. On day 16, the rats underwent electrode implantation into the VTA and allowed to recover for 9 days. Rats were filmed at day 10 after surgery, and again after AES treatment. After this they were filmed once a week throughout the month following the AES treatment (Janssen et al, 1960; Kazlauckas et al, 2005; Malkesman et al, 2007; Strekalova et al, 2004).

Anhedonia test (sucrose self-administration). The experiment was designed to test whether AES increases basic reward behavior ('anhedonia': used as a measure of depressive-like behavior; De La et al, 2004, 2005). Rats were transferred daily into operant conditioning chambers (Med-Associates Inc.; St Albans, Vermont) for $30 \mathrm{~min}$ sessions, during their dark cycle, and allowed to selfadminister sucrose $(10 \%$ sucrose solution; $0.13 \mathrm{ml}$ per infusion) delivered into a liquid drop receptacle for oral consumption. During the infusion, a light located above the active lever was lit for $20 \mathrm{~s}$. During the $20 \mathrm{~s}$ intervals of sucrose infusion, active lever presses were recorded, but no additional sucrose reinforcement was provided. Presses on the inactive lever were recorded, but they did not activate the infusion pump and light. Rats were returned to the home cages at the end of the daily session. Animals had free access to water and food, ensuring that the test measured only anhedonic behavior.

Water self-administration. A separate group of rats was trained to bar press for water reinforcement. Rats were allowed $15 \mathrm{ml}$ of water per day in addition to approximately $4.0 \mathrm{ml}$ of water consumed during the daily sessions. The operant chambers, reinforcement schedule, and session duration were identical to those used for sucrose selfadministration. Rats received $0.13 \mathrm{ml}$ of water per lever press, delivered into a drinking dish in the operant chamber. The number of active lever responses, reinforcements, and inactive lever responses were recorded.

Novelty interest test. Rats were allowed to explore a new object for $5 \mathrm{~min}$ in a plastic polymer box $(90 \times 90 \times 30 \mathrm{~cm})$. Illumination intensity was $5 \mathrm{~lx}$. The object, with a complex texture surface (artificial camel, $15 \times 6 \times 5 \mathrm{~cm}$ ), was fixed to the near side of the exploration area. The total duration of time spent exploring the object was scored (Kazlauckas et al, 2005; Strekalova et al, 2004).

Battery sequence. Figure 1 describes the flow chart of experimental procedures. Owing to the high amount of stress involved in the behavioral swim test, we chose to use separate groups of rats to assess their performance in this category. Naive FSL and SD rats were used as controls. In addition, separate FSL groups were used for the antidepressant, ECT and AES treatments in comparison with sham-operated rats. The swim test was performed immediately after AES or sham AES, thereafter rats were monitored weekly by the swim test.

For each separate group of rats we performed the following tests: the sucrose test, social behavior test, locomotor activity test, and the novelty exploration test.

The social behavior and locomotor activity behavioral tests were performed on naive rats on separate occasions. After each of these two tests (6 h later), the sucrose selfadministration task was performed. The initial period was 




Figure I Sequence of behavior tests.

dedicated to training and habituation (2 weeks), and following this, a behavioral baseline was measured.

After establishing a behavioral baseline, an electrode was implanted into the VTA. Following a period of rehabilitation ( 2 weeks), the rats were subjected once again to the behavioral battery described above. The results obtained before the AES served as a sham-operated control.

Next, the rats underwent AES treatment, following which their performance in the above-mentioned behavioral tests was evaluated. The first test in the series was conducted within $10 \mathrm{~min}$ of the end of the AES treatment. The rats' performance in these tests continued to be monitored weekly throughout a period of 4 weeks, to determine the longitudinal effect of AES treatment.

The novelty interest test was performed once, 4 weeks after AES on separate groups of rats, to preserve the novelty of the task. As controls for this task, we used naive SD, sham-operated SD, naive FSL, and sham-operated FSL.

Antidepressant administration. Antidepressants were prepared and administered i.p. daily for 7, 9, or 14 days to FSL rats as previously described (Dremencov et al, 2006).

Histology. At the conclusion of the experiment, animals were anesthetized and transcardially perfused with PBS $\times 1$ followed by $4 \%$ paraformaldehyde. The brains were removed and immersed in $4 \%$ paraformaldehyde for $24 \mathrm{~h}$, and then in phosphate buffer with $30 \%$ sucrose for $48 \mathrm{~h}$. The brains were then frozen on dry ice and sliced $(40 \mu \mathrm{m}$ sections) using a cryostat microtome. Sections were mounted on glass slides (coated with $2 \%$ gelatin), stained with Cresyl violet, and subsequently examined under a microscope to verify the placement of the electrode. Only animals in which localization of the electrode had been confirmed were included in the study.

Brain dissection and extraction. Rats were decapitated and their brains were removed rapidly 30 days after AES. The entire hypothalamus was surgically dissected and frozen immediately in liquid nitrogen. The brains were then placed in a rat brain mold (constructed at Bar-Ilan University) on ice, and serial $0.5-\mathrm{mm}$ sections were cut and placed on chilled microscope slides. Tissue punches were taken rapidly, using a stainless steel cannula with an inner diameter of $1.1 \mathrm{~mm}$, according to the following coordinates: A1.5-2.0 mm for the N.Acs, prefrontal cortex (PFC); P5.5$5.0 \mathrm{~mm}$ VTA (Dremencov et al, 2005), and included most of the desired regions. The tissue samples were frozen immediately in liquid nitrogen and stored at $-70^{\circ} \mathrm{C}$ until extraction for mRNA or protein analysis.

Reverse transcription and quantitative real-time RT-PCR. Total RNA was extracted using a Stratagene absolute RNA miniprep kit including DNAse treatment procedure (Stratagene, 400800). A total of $0.5 \mu \mathrm{g}$ RNA was reversetranscribed according to manufacturer's instructions (Stratascript 5.0 multi-temp RT kit; Stratagene), using oligo-dT $15 \mathrm{bp}$ primers (Promega, La Jolla, CA; C1101). The relative expression of the investigated genes was measured by realtime quantitative PCR on an $\mathrm{M} \times 3000 \mathrm{P}$ detection system (Stratagene) using the Dynamo HS SYBR Green master kit (Finnzymes, FZ-F-410L). Quantitative PCR reactions were 
carried out in triplicate and the data were analyzed with the comparative $C_{\mathrm{T}}$ method using MX PRO software (Stratagene). Primers were designed using Primer3 software (http://frodo.wi.mit.edu/). Product specificity was confirmed in the initial experiments by agarose gel electrophoresis and routinely by melting curve analysis. The BDNF primers were based on a coding sequence of the rat $B D N F$ gene: upstream: 5'-GCGGCAGATAAAAAGACTGC-3' and downstream: $5^{\prime}$-GCCAGCCAATTCTCTTTTTG- ${ }^{\prime}$. As an endogenous normalization factor, the transcript levels of the HPRT gene were used: upstream: 5'-AGGCCAGACTTT GTTGGATT- $3^{\prime}$ and downstream: $5^{\prime}$-GCTTTTCCACTTTCGC TGAT-3'.

Western blot analysis. Tissues samples were thawed and homogenized in lysis buffer $(150 \mathrm{mM} \mathrm{NaCl}, 5 \mathrm{mM}$ EDTA, $1 \%$ Nonidet P- $40,0.5 \%$ sodium deoxycholate, $1 \mathrm{mM} \mathrm{Na}_{2} \mathrm{VO}_{4}$, $200 \mathrm{mM}$ HEPES, and $50 \mathrm{mM} \mathrm{NaF}$ ) with Complete Mini Protease Inhibitor Cocktail Tablets (Roche, Germany). Solutions were incubated for $30 \mathrm{~min}$ at $4^{\circ} \mathrm{C}$. The proteins were cleared by centrifugation at $14000 \mathrm{~g}$ for $20 \mathrm{~min}$ at $4^{\circ} \mathrm{C}$. Protein concentration was determined by BCA kit (Pierce, Rockford, USA). Protein $(30 \mu \mathrm{g})$ from each sample was resolved in SDS-PAGE. Electroblotted proteins were detected using antibodies: anti-BDNF and anti-actin all from Santa Cruz (Santa Cruz, CA, USA) bound antibodies were visualized using a chemiluminescence reaction (Pierce).

\section{Statistics}

All data are expressed as mean \pm standard error of the mean (SEM). Significance was determined by an independent Student's $t$-test or ANOVA, and $p<0.05$ was considered significant. Variance data are expressed as variance \pm confidence value. Significance of variances was determined by an F-test, and $p<0.05$ was considered significant. Correlation of parameters was determined by Pearson's test.

All animal procedures were approved by the Bar-Ilan University Animal Care Committee and were carried out in accordance with the National Institutes of Health Guide for the Care and Use of Laboratory Animals.

\section{RESULTS}

\section{Analysis of VTA Neuronal Firing: Estimation Patterns for Acute Electrical Stimulation}

In SD rats, the average firing was calculated to be five spikes per burst with an $80-160 \mathrm{~ms}$ interval between spikes. FSL rats were unable to fire at the same rate as controls, however they compensate for this inability by firing an increased number of small bursts calculated to be three spikes per burst with an $80-160 \mathrm{~ms}$ interval between spikes (Figure 2; two-way ANOVA (strains $\mathrm{F}(1,11)=33.7, p<0.001$; burst type $\mathrm{F}(1,11)=53.4, p<0.001$; interactions $\mathrm{F}(1,11)=55.4$, $p<0.001)$ ). The characteristics of these patterns were established previously (Friedman et al, 2008).

We hypothesized that DBS may function through correcting specific electrophysiological patterns. Thus, we constructed a defined electrical template from the recorded and analyzed SD VTA cell-firing data. Next, using AES, we applied this pattern of stimulation, fashioned to mimic the

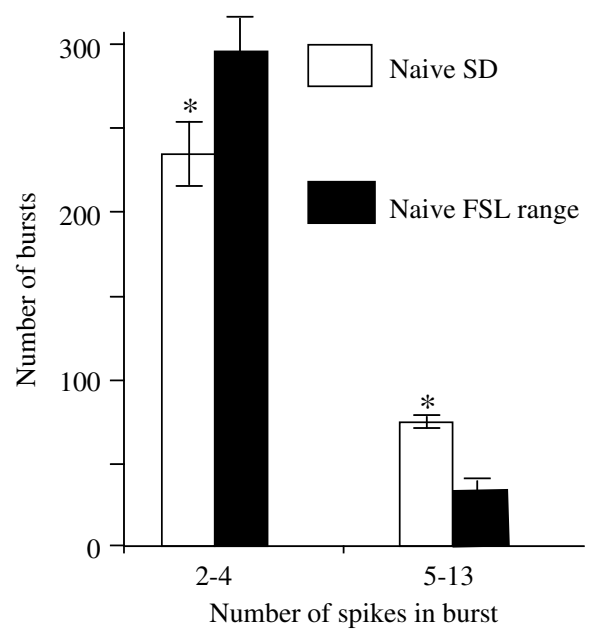

Figure 2 Analysis of ventral tegmental area (VTA) electrophysiology. Electrodes were inserted into the VTA and the firing of dopamine-like cells was recorded. Panel illustrates the burst distribution histogram in SpragueDawley vs Flinder Sensitive Line (FSL) rats ( $N=12,2500$ spike recordings). Two-way ANOVA (strains $F(I, I I)=33.7, p<0.00 I$; burst type $F(I, \mid I)=53.4, \quad p<0.00 I$, interactions $F(I, \mid I)=55.4, \quad p<0.00 I)$. * $p<0.00$ I vs corresponding group.

firing pattern of the normal rat, to the FSL VTA (see Supplementary Figure 1A).

\section{Normalization of FSL Depressive-Like Behavior After Acute Electrical Stimulation}

Rats were trained for behavioral tasks and a baseline was recorded. A concentric bipolar electrode was implanted into the VTA of FSL and control rats, and 2 weeks later depressive-like manifestations were monitored using a battery of behavioral tests. Then, FSL rats received AES through the electrode, and depressive-like behavioral manifestations were again monitored using the same battery of behavioral tests. An improvement in all measured parameters of depressive-like behavior was observed, which persisted for at least 4 weeks (Figures 3-5).

Treating FSL rats with AES resulted in enhanced activity during stimulation, measured as rearing time, grooming, eating, and drinking (700.4 $\pm 4.9 ; 10.36 \pm 3.31 ; 70 \pm 15$; $15 \pm 5 \mathrm{~s}$ per $20 \mathrm{~min}$ monitoring, respectively) as compared to minimal activity $(<1 \pm 1 \mathrm{~s}$ per $20 \mathrm{~min}$ monitoring in all parameters measured) of nontreated FSL rats $(N=25$ animals in each group).

The rats' level of despair was tested by measuring immobility in the swim test. Because this test is traumatic and stressful, the results of the AES treatment were compared to sham-operated and naive SD rats (nondepressive normal rats), naive FSL rats, and FSL rats treated with chemical antidepressants or ECT (see Figure $3 a$ and $b$ ). We found that immobility of depressive rats was higher than in controls, whereas treatment with antidepressants, ECT, or AES normalized the degree of immobility in FSLs (Figure 3) (antidepressants, $N=20$ per group; AES, $N=14$; sham, $N=15$; control groups, $N=10 ; p<0.05$ vs naive range; single-factor ANOVA). However, AES showed a significant faster onset and long-term effect compared to other treatment. Stimulation of FSL rat brain in a nonspecific 


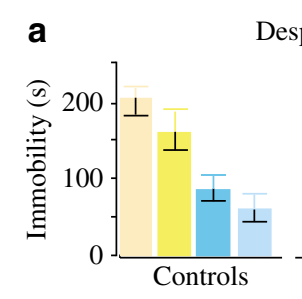

Despair behavior test (swim test)
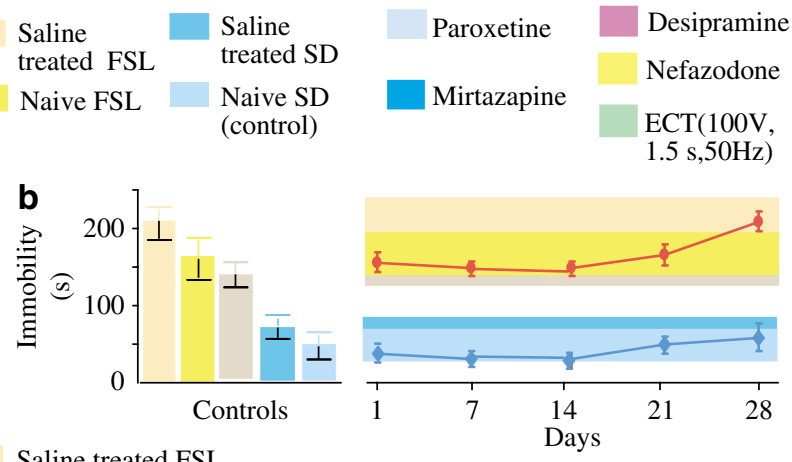

Saline treated FSL

Naive FSL

AES (Deep Mes Nu) treated FSL

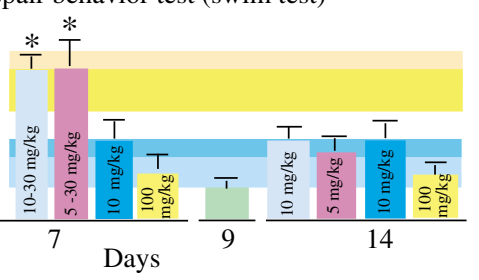

Nefazodone

ECT $(100 \mathrm{~V}$,

\section{.}



Figure 3 Effect of conventional antidepressant treatments and acute electrical stimulation (AES) on motivation. (a) Effect of antidepressants was measured by immobility of Flinder Sensitive Line (FSL) rats in a modified Porsolt test (see Materials and methods). Measurements were conducted 7,9 , and 14 days after treatment with the various antidepressants $(N=10$ per group). Means of control groups are demonstrated as bands extending across the panel, displayed according to their respective column color, to facilitate comparison to treatment groups. $* p<0.05$ vs normal range. (b) Effect of AES as measured by immobility of FSL rats in the swim test referred to above. Measurements were conducted I, 7, I4, 2I, and 28 days after treatment with AES (deep brain stimulation (DBS), $N=14$; sham, $N=15 ; 250-310 \mathrm{~g}$ rats single-factor ANOVA: $F(1,26)=40.02, p<10^{-6}$ ). Means of control groups are also demonstrated as bands extending across the panel, displayed according to their respective column color, to facilitate comparison to treatment groups. (c) Effect of AES on locomotor activity in a novel open field (serves as a control for the swim test). Measurements (distance moved) were conducted for each individual animal at the following states: naive, sham-operated, and -treated (I, 7, 14, 21, and 28 days after treatment with AES). ANOVA did not reveal significance.

region, the deep mesencephalic nucleus, resulted in no effect on depressive behavior, measured by a swim test conducted immediately after the session of stimulation $(142 \pm 14.02 v s \quad 162.2 \pm 13.24 \mathrm{~s}$ in FSL and sham-operated rats). No effect was seen even seven days after stimulation (144.78 \pm 14.18 and $153.6 \pm 9.49 \mathrm{~s}$; FSL $v s$ sham operated rats).

The observed decrease in immobility following AES can have two possible causes: AES to the VTA may have acted as
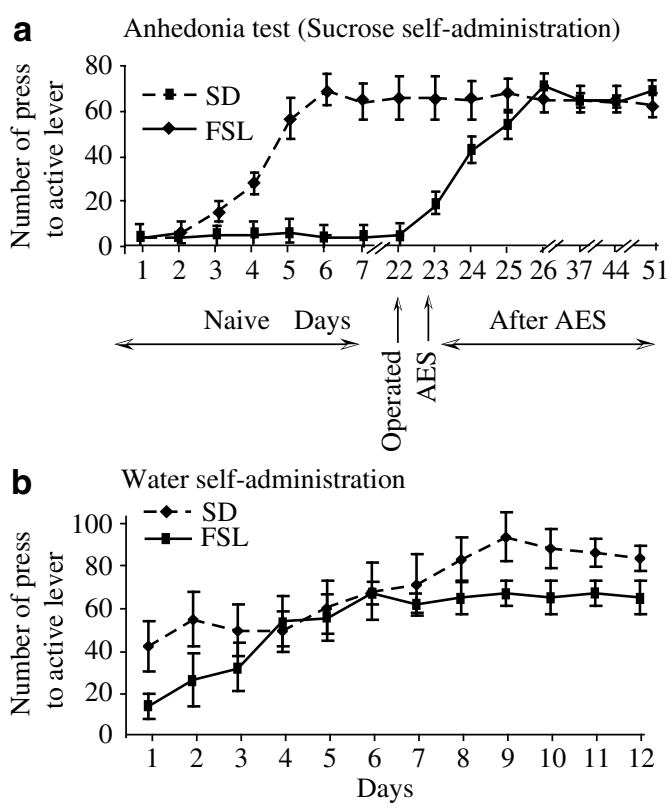

Figure 4 Effect of acute electrical stimulation (AES) on anhedonia. (a) The effect of AES treatment was measured by the number of active lever presses in the sucrose self-administration test ( $N=10$ per group). (b) Water self-administration of Flinder Sensitive Line (FSL) rats (serves as a control for the anhedonia test). $N=10$ per group. ANOVA did not reveal significance.

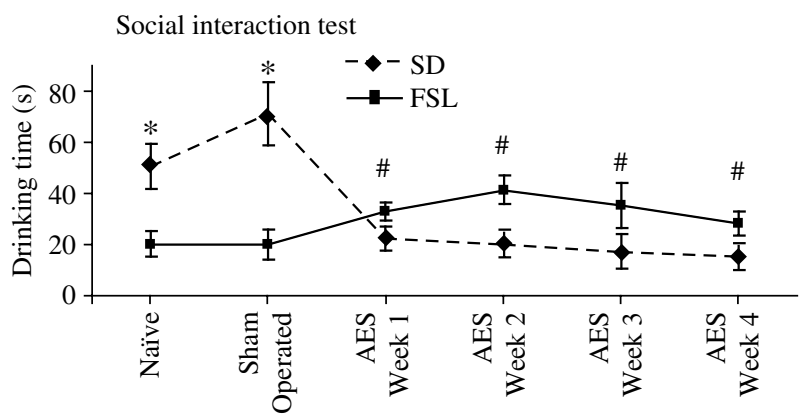

Figure 5 Effect of acute electrical stimulation (AES) on social interaction. Social interaction was measured using the dominant-submissive test. Time spent in the feeder zone (milk with $20 \%$ sucrose) by each animal was recorded during a 5-min testing period. Measurements were conducted for naive rats, operated rats, rats I day after AES and on the second, third, and fourth week after AES (Sprague-Dawley (SD) rats, $N=10$; Flinder Sensitive Line (FSL) rats, $N=8$; * $p<0.05$; repeated-measures ANOVA).

an antidepressant, or it may have had a stimulant effect similar to amphetamine, for example. To examine these two options, we measured the locomotor activity of rats in the open-field test (Figure 3c). Naive FSL rats have lower locomotor activity than the control SD rats, and treatment with AES did not improve locomotor activity. This result supports the notion that AES has an antidepressant, and not stimulant, function (SD rats, $N=10$; FSL rats, $N=8$; $p<0.05$; repeated-measures ANOVA).

Anhedonia was measured using a sucrose self-administration test. FSL rats exhibited anhedonic-like behavior, measured by a low number of presses on the active lever as compared to controls. Following treatment with AES, FSL 


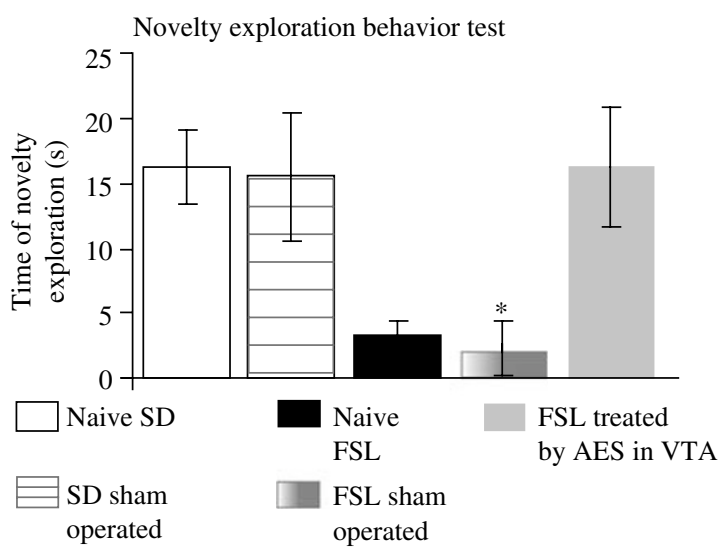

Figure 6 Effect of acute electrical stimulation (AES) on novelty exploration. Time exploring of a new object by each animal was recorded during a 5-min testing period. Measurements were conducted for five different animal groups: Naive Sprague-Dawley (SD) rats, sham-operated SD rats, naive Flinder Sensitive Line (FSL) rats, sham-operated FSL rats, and AES-treated FSL rats, 4 weeks after treatment (SD rats, $N=10$; FSL rats, $N=8 ; * 0<0.05$; repeated-measures ANOVA)

rats began to learn the self-administration task. After 1 week, AES-treated FSL and control SD groups showed an equal number of lever presses (Figure 4a) (SD rats, $N=10$; FSL rats, $N=8$ ).

The disrupted operant behavior of FSL rats in the sucrose self-administration test may be due to a memory deficit, physical low performance, or because of their high levels of anhedonia. To examine these possibilities, we measured water self-administration following water deprivation in FSL and control rats (Figure 4b). Naive FSL rats did not show significant differences in active lever responses for water reinforcement as compared to controls (SD rats, $N=10$; FSL rats, $N=10$ ). This shows that the effects achieved by DBS are only relevant to tasks that involve hedonic motivation and they do not entail a learning deficit or any other impairment of physical performance.

Social interaction was measured by the dominantsubmissive test. Before treatment, FSL rats showed submissive behavior compared to SD rats, whereas following AES treatment, an inverse relationship was found (Figure 5) (SD rats, $N=10$; FSL rats, $N=8$; $p<0.05$; repeatedmeasures ANOVA). The results of this test are based on the comparison between the behavior of one rat to the other. Accordingly, the shift in the dominant-submissive relationship was manifested by an increase in competition by FSL rats for access to the feeder leading to a decline in the drinking time of the SD rats.

Novelty (seeking) exploration behavior was measured in an open field, allowing rats to explore a new object. FSL rats show a low level of 'interest' in the new object, whereas AES treatment normalized object exploration (Figure 6) (SD rats, $N=10$; FSL rats, $N=8 ; p<0.05$; repeated-measures ANOVA).

\section{BDNF Expression in the Brain's Regions}

Rats were treated with intra-VTA AES, and BDNF mRNA and protein levels were subsequently measured in brain regions related to motivation and hedonia (VTA, N.Ac, and PFC in sham-treated SD, sham-treated FSL, and AES-treated
FSL rats. In the VTA, no significant differences were found between BDNF mRNA and protein levels of the three groups (Figure 7a and $\mathrm{d}$ ). In the N.Ac, lower BDNF mRNA and protein levels were observed in untreated FSL rats (though not significant for mRNA levels and significant for protein levels (one-way ANOVA $\mathrm{F}(2,14)=3.7,{ }^{\star} p<0.002$ )), which were normalized by intra-VTA AES stimulation (Figure $7 \mathrm{~b}$ and e). In the PFC, FSL rats had significantly lower BDNF mRNA levels as compared to SD rats (one-way ANOVA, $\mathrm{F}(2,9)=6.44, p<0.005)$, which were normalized by AES treatment (Figure $7 \mathrm{c}$ ) and significantly lower BDNF protein levels (one-way ANOVA $\mathrm{F}(2,14)=6.37, p<0.005$ ) normalized by AES treatment (Figure 7f).

\section{DISCUSSION}

Previously, we found that the dopaminergic system is important in the depressive symptoms of FSL rats. These symptoms were normalized using various clinically employed antidepressants (Dremencov et al, 2006; Overstreet et al, 2005) in parallel to stabilization of dopaminergic system functionality (Friedman et al, 2005, 2007, 2008; Overstreet et al, 2005).

In a recent study (Friedman et al, 2008), we recorded and analyzed the firing pattern of dopaminergic cells in the VTA. We found that the VTA of SD rats has the capability of firing bursts with a large amount of spikes, whereas FSL rats rarely show this pattern. Thus, we constructed a defined electrical template from the recorded and analyzed SD VTA cell-firing data. In the present study, using AES, we applied this pattern of stimulation, fashioned to mimic the firing pattern of the normal rat, in the VTA of the FSL rats. Previous studies have demonstrated that similar parameters of pulse duration and intensity to those employed in our unique pattern of stimulation were successful in activating VTA DA neurons and consequentially inducing DA release in the N.Ac and PFC (Fiorino et al, 1993; Gessa et al, 2000). The results of this experiment demonstrated that AES has an unexpectedly long-term effect on behavioral symptoms in FSL rats (as opposed to the need for continuous DBS in the treatment of Parkinson's disease and Tourette's syndrome). This finding may be due to the biphasic nature of depression, in which the depressed individual alternates between normal, nondepressive and abnormal 'depressive' behavioral stages. This pattern follows the dynamic fluctuation mode of neuronal activity in the normal brain (Friedman et al, 2008). AES may restore FSL neuronal dynamics to their normal stable mode.

When comparing AES treatment to other pharmacological treatments, we were able to demonstrate that AES has several advantages, namely, fast onset of action, a selective effect within specific brain sites, and greater efficacy in correcting depressive behavior (Figures 4-7). Specifically, we show that AES exerts its effect immediately within one short treatment session, whereas antidepressants or ECT require between 7 and 14 treatment sessions. The remedial effect of AES persists for up to 1 month after a single stimulation. Stimulation of FSL rat brain in the deep mesencephalic nucleus (a nonspecific region which is not involved in motivation and emotion) resulted in no effect on depressive behavior. Furthermore, it is important to note 


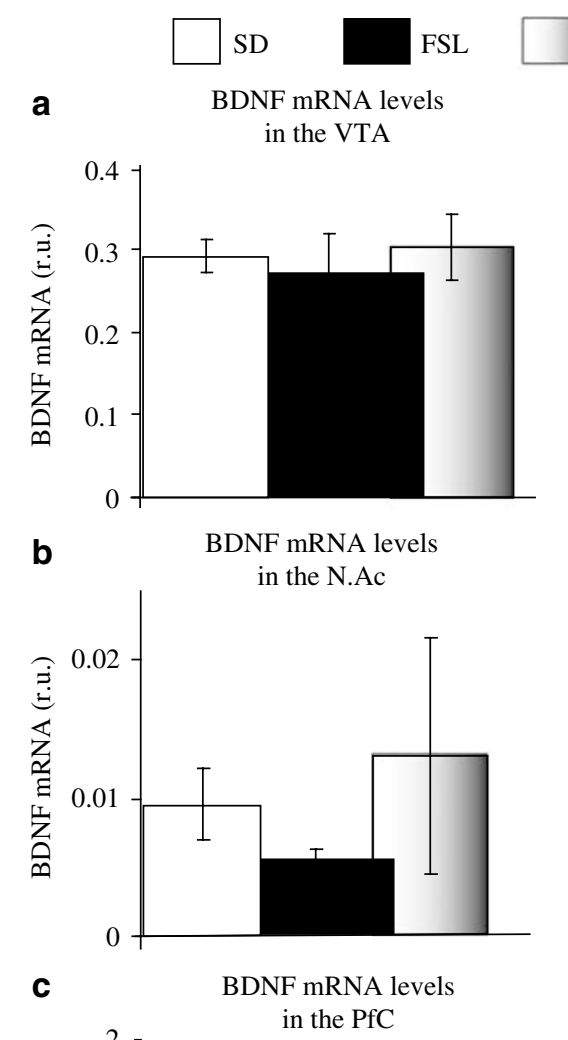

FSL treated with AES in VTA
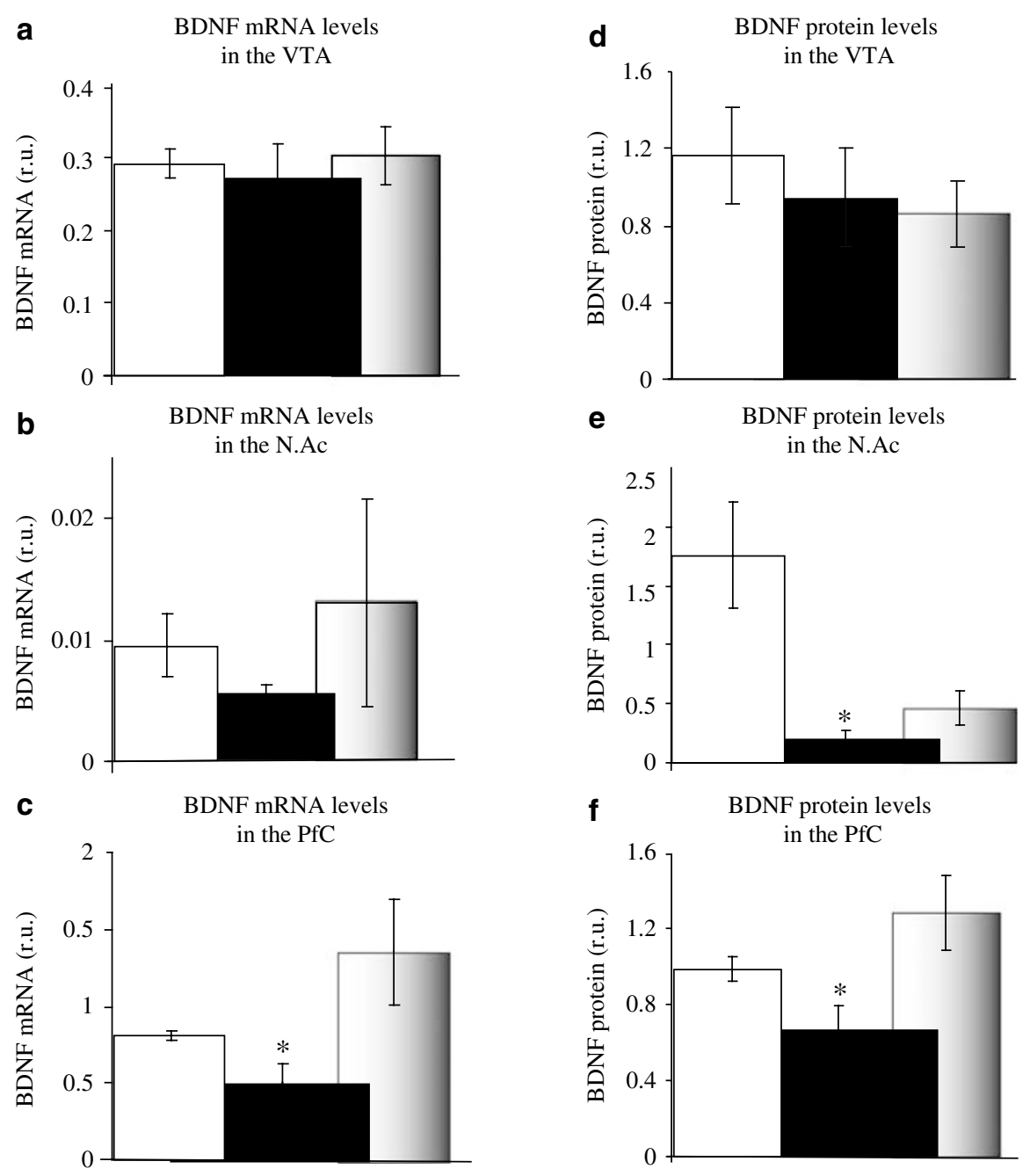

Figure 7 Levels of brain-derived neurotrophic factor (BDNF) transcript and protein in different brain regions. Tissue punches were taken from Flinder Sensitive Line (FSL), Sprague-Dawley (SD), and acute electrical stimulation (AES)-treated FSL rats. BDNF mRNA levels were analyzed by real-time PCR and demonstrated in the ventral tegmental area (VTA) (a), N.Ac (b), and PFC (one-way ANOVA F $(2,9)=6.44$, * p <0.005) (c). Results are expressed as relative units (r.u.), a number resulting from the normalization procedure. BDNF protein levels were analyzed by Western blot Analysis and demonstrated in the VTA (d), N.Ac (one-way ANOVA F $(2,14)=3.7$, * $p<0.002$ ) (e), and PFC (one-way ANOVA F $(2,14)=6.37$, * $p<0.005$ ) (f). Results are expressed as relative units (r.u.), a number resulting from the normalization procedure.

that we used specific parameters of electrical stimulation that have a localized effect of no more than $700-800 \mu \mathrm{m}$ from the tip of the electrode (Roger Lemon Methods for Neuronal Recording in Conscious Animals, page 40 Figure 2.11). Thus, AES treatment is highly regional specific.

A possible limitation confronting the future implementation of this procedure would be the sensitive location of the VTA. The VTA is situated deep within the interior of the brainstem and as a result the ability to gain access to this area might prove to be somewhat of a challenge. This being said, we would like to note that within the framework of our limited experiment we were able to improve our technique of electrode implantation and stimulation to such a degree that nearly all animals succeed in recovering from the procedure ( 2 of 20 animals succumbed to the surgical process but this was likely due to anesthesia complications). Furthermore, in light of the physiological relationship between the VTA and oculomotor functions (Wylie et al,
2005), it is important to note that no ocular abnormalities were observed. There is of course a need to be cautious in drawing inference from our success in performing the procedure in an animal model to the possibility of succeeding similarly with human subjects.

However, we suggest that similar to the clinically performed DBS treatment of Parkinson's disease (Limousin and Martinez-Torres, 2008) and depression (Schlaepfer et al, 2008), in which similarly difficult regions of the brain (such as the subthalamic nucleus, the globus pallidus pars interna, and the N.Acs) are accessed with success, that future improvement of surgical technique and stimulation technology and the accurate definition of the optimal site and angle of entry may lead to the advancement of this procedure as a feasible treatment option.

Evidence implicating BDNF in the mechanism of action of antidepressants includes the findings that chronic, but not acute, treatment with the serotonergic antidepressant 
fluoxetine, the noradrenergic antidepressant desipramine, increase BDNF expression in the brain (Thome et al, 2000). We demonstrated that AES significantly normalizes BDNF mRNA levels in the PFC, and also in the N.Ac (nonsignificant). However, in the VTA, no differences were found between groups. This indicates that BDNF transcription levels are correlated with depressive-like behavior and are not a result of neuronal lesion or electrical shock. Therefore, a functional role for BDNF in mediating antidepressant effects of AES is suggested.

Owing to the preliminary nature of these results additional research shall be required to determine whether this is a viable and practical mode of treatment. But in light of the current findings we feel that it holds much promise and potential as a possible treatment of major depression.

\section{ACKNOWLEDGEMENTS}

We thank Dr Tamar Green-Sadan and Ms Anita Ulmann for critically reviewing the paper.

\section{DISCLOSURE/CONFLICTS OF INTEREST}

All the authors state that they have no conflict of interest to declare. Patent number 60/846.345 - the invention generally pertains to deep brain stimulation with a selected pattern of the ventral tegmental area as a new form of treatment for chronic depression.

\section{REFERENCES}

Barde YA, Edgar D, Thoenen H (1982). Purification of a new neurotrophic factor from mammalian brain. EMBO J 1: 549-553.

De La GR, Asnis GM, Fabrizio KR, Pedrosa E (2005). Acute diclofenac treatment attenuates lipopolysaccharide-induced alterations to basic reward behavior and HPA axis activation in rats. Psychopharmacology (Berl) 179: 356-365.

De La GR, Fabrizio KR, Radoi GE, Vlad T, Asnis GM (2004). The non-steroidal anti-inflammatory drug diclofenac sodium attenuates lipopolysaccharide-induced alterations to reward behavior and corticosterone release. Behav Brain Res 149: 77-85.

Dias BG, Banerjee SB, Duman RS, Vaidya VA (2003). Differential regulation of brain derived neurotrophic factor transcripts by antidepressant treatments in the adult rat brain. Neuropharmacology 45: 553-563.

Dremencov E, Gispan-Herman I, Rosenstein M, Mendelman A, Overstreet DH, Zohar J et al (2004). The serotonin-dopamine interaction is critical for fast-onset action of antidepressant treatment: in vivo studies in an animal model of depression. Prog Neuropsychopharmacol Biol Psychiatry 28: 141-147.

Dremencov E, Newman ME, Kinor N, Blatman-Jan G, Schindler CJ, Overstreet DH et al (2005). Hyperfunctionality of serotonin-2C receptor-mediated inhibition of accumbal dopamine release in an animal model of depression is reversed by antidepressant treatment. Neuropharmacology 48: 34-42.

Dremencov E, Weizmann Y, Kinor N, Gispan-Herman I, Yadid G (2006). Modulation of dopamine transmission by 5HT2C and 5HT3 receptors: a role in the antidepressant response. Curr Drug Targets 7: 165-175.

Fiorino DF, Coury A, Fibiger HC, Phillips AG (1993). Electrical stimulation of reward sites in the ventral tegmental area increases dopamine transmission in the nucleus accumbens of the rat. Behav Brain Res 55: 131-141.
Friedman A, Deri I, Friedman Y, Dremencov E, Goutkin S, Kravchinsky E et al (2007). Decoding of dopaminergic mesolimbic activity and depressive behavior. J Molec Neurosci 32: 72-79.

Friedman A, Dremencov E, Crown H, Levy D, Mintz M, Overstreet $\mathrm{DH}$ et al (2005). Variability of the mesolimbic neuronal activity in a rat model of depression. Neuroreport 16: 513-516.

Friedman A, Friedman Y, Dremencov E, Yadid G (2008). VTA dopamine neuron bursting is altered in an animal model of depression and corrected by desipramine. J Mol Neurosci 34: 201-209.

Gershon AA, Vishne T, Grunhaus L (2007). Dopamine D2-like receptors and the antidepressant response. Biol Psychiatry 61: $145-153$.

Gessa GL, Devoto P, Diana M, Flore G, Melis M, Pistis M (2000). Dissociation of haloperidol, clozapine, and olanzapine effects on electrical activity of mesocortical dopamine neurons and dopamine release in the prefrontal cortex. Neuropsychopharmacology 22: 642-649.

Grace AA, Bunney BS (1984a). The control of firing pattern in nigral dopamine neurons: burst firing. J Neurosci 4: 2877-2890.

Grace AA, Bunney BS (1984b). The control of firing pattern in nigral dopamine neurons: single spike firing. J Neurosci 4: 28662876.

Janssen PA, Jageneau AH, Schellekens KH (1960). Chemistry and pharmacology of compounds related to 4-(4-hydroxy-4-phenylpiperidino)-butyrophenone. IV. Influence of haloperidol ( $R$ 1625) and of chlorpromazine on the behaviour of rats in an unfamiliar 'open field' situation. Psychopharmacologia 1: 389-392.

Kapur S, Mann JJ (1992). Role of the dopaminergic system in depression. Biol Psychiatry 32: 1-17.

Kazlauckas V, Schuh J, Dall'Igna OP, Pereira GS, Bonan CD, Lara DR (2005). Behavioral and cognitive profile of mice with high and low exploratory phenotypes. Behav Brain Res 162: 272-278.

Kessler RC, Berglund P, Demler O, Jin R, Koretz D, Merikangas KR et al (2003). The epidemiology of major depressive disorder: results from the National Comorbidity Survey Replication (NCSR). JAMA 289: 3095-3105.

Knapp RJ, Goldenberg R, Shuck C, Cecil A, Watkins J, Miller C et al (2002). Antidepressant activity of memory-enhancing drugs in the reduction of submissive behavior model. Eur J Pharmacol 440: 27-35.

Limousin P, Martinez-Torres I (2008). Deep brain stimulation for Parkinson's disease. Neurotherapeutics 5: 309-319.

Malatynska E, Kostowski W (1984). The effect of antidepressant drugs on dominance behavior in rats competing for food. Pol J Pharmacol Pharm 36: 531-540.

Malatynska E, Rapp R, Crites G (2002). Dominant Behavior Measured in a Competition Test as a Model of Mania, International Behavioral Neuroscience Society Meeting (IBNS), Capri, Italy.

Malkesman O, Braw Y, Weller A (2007). Assessment of antidepressant and anxiolytic properties of NK1 antagonists and substance $\mathrm{P}$ in Wistar Kyoto rats. Physiol Behav 90: 619-625.

Mayberg HS, Lozano AM, Voon V, McNeely HE, Seminowicz D, Hamani C et al (2005). Deep brain stimulation for treatmentresistant depression. Neuron 45: 651-660.

Naranjo CA, Tremblay LK, Busto UE (2001). The role of the brain reward system in depression. Prog Neuropsychopharmacol Biol Psychiatry 25: 781-823.

Nibuya M, Morinobu S, Duman RS (1995). Regulation of BDNF and trkB mRNA in rat brain by chronic electroconvulsive seizure and antidepressant drug treatments. J Neurosci 15: 7539-7547.

Ordway GA, Mann JJ (2002). Neurocircuitry of mood disorders. In: Davis KL, Coyle JT, Nemeroff C (eds). Neuropsychopharmacology: The Fifth Generation of Progress. Lippincott Williams \& Wilkins: Philadelphia. pp 1051-1064. 
Overstreet DH (1993). The Flinders Sensitive Line rats: a genetic animal model of depression. Neurosci Biobehav Rev 17: 51-68.

Overstreet DH, Friedman E, Mathe AA, Yadid G (2005). The Flinders Sensitive Line rat: a selectively bred putative animal model of depression. Neurosci Biobehav Rev 29: 739-759.

Pinhasov A, Crooke J, Rosenthal D, Brenneman D, Malatynska E (2005a). Reduction of submissive behavior model for antidepressant drug activity testing: study using a video-tracking system. Behav Pharmacol 16: 657-664.

Pinhasov A, Ilyin SE, Crooke J, Amato FA, Vaidya AH, Rosenthal D et al (2005b). Different levels of gamma-synuclein mRNA in the cerebral cortex of dominant, neutral and submissive rats selected in the competition test. Genes Brain Behav 4: 60-64.

Reddy PL, Khanna S, Subhash MN, Channabasavanna SM, Rao BS (1992). CSF amine metabolites in depression. Biol Psychiatry 31: $112-118$.
Schlaepfer TE, Cohen MX, Frick C, Kosel M, Brodesser D, Axmacher $\mathrm{N}$ et al (2008). Deep brain stimulation to reward circuitry alleviates anhedonia in refractory major depression. Neuropsychopharmacology 33: 368-377.

Strekalova T, Spanagel R, Bartsch D, Henn FA, Gass P (2004). Stress-induced anhedonia in mice is associated with deficits in forced swimming and exploration. Neuropsychopharmacology 29: 2007-2017.

Thome J, Sakai N, Shin K, Steffen C, Zhang YJ, Impey S et al (2000). cAMP response element-mediated gene transcription is upregulated by chronic antidepressant treatment. J Neurosci 20: 4030-4036.

Wylie DR, Ogilvie CJ, Crowder NA, Barkley RR, Winship IR (2005). Telencephalic projections to the nucleus of the basal optic root and pretectal nucleus lentiformis mesencephali in pigeons. Vis Neurosci 22: 237-247.

Supplementary Information accompanies the paper on the Neuropsychopharmacology website (http://www.nature.com/npp) 